\title{
157nm Pellicles: Polymer Design for Transparency and Lifetime
}

\author{
Roger H. French ${ }^{1}$, Robert C. Wheland ${ }^{1}$, Weiming Qiu ${ }^{1}$, M. F. Lemon ${ }^{1}$, Gregory S. Blackman ${ }^{1}$, Edward \\ Zhang $^{2}$, Joseph Gordon ${ }^{2}$, Vlad Liberman ${ }^{3}$, Andrew Grenville ${ }^{4}$, Rod Kunz ${ }^{3}$, Mordechai Rothschild ${ }^{3}$ \\ 1. DuPont Co. Central Research, E356-384, Wilmington DE 19880-0356. \\ 2. DuPont Photomasks Inc. 4 Finance Dr., Danbury CT, 06810 \\ 3. Lincoln Laboratory, Massachusetts Institute of Technology, Lexington MA 02420 \\ 4. Intel Corporation / International Sematech
}

Keywords: $157 \mathrm{~nm}$ lithography, pellicle, fluoropolymer, absorbance, radiation durability, VUV spectroscopy, VUV ellipsometry

\section{ABSTRACT}

The introduction of $157 \mathrm{~nm}$ as the next optical lithography wavelength has created a need for new soft (polymeric) or hard (quartz) pellicle materials. Pellicles should be $>98 \%$ transparent to incident $157 \mathrm{~nm}$ light and, ideally, sufficiently resistant to photochemical damage to remain useful for an exposure lifetime of $7.5 \mathrm{~kJ} / \mathrm{cm}^{2}$.

The transparency specification has been met. We have developed families of experimental Teflon ${ }^{\mathrm{TM}} \mathrm{AF}(\mathrm{TAFx})$ polymers with $>98 \%$ transparency which can be spin coated and lifted as micron-scale, unsupported membranes. Still higher transparencies should be possible once optimization of intrinsic (composition, end groups, impurities, molecular weight) and extrinsic (oxygen, absorbed hydrocarbons, contaminants) factors are completed. The measured transparencies of actual pellicle films, however, are affected by many factors other than absorption. Film thickness must be precisely controlled so as to allow operation at the fringe maxima for the lithographic wavelength. Roughness and thickness uniformity are also critical. An important part of our program has thus been learning how to spin membranes from the solvents that dissolve our pellicle candidates.

Meeting the durability specification at $157 \mathrm{~nm}$ remains a major concern. The $157 \mathrm{~nm}$ radiation durability lifetime of a polymer is determined by two fundamental properties: the fraction of $157 \mathrm{~nm}$ radiation absorbed and the fraction (quantum efficiency) of this absorbed radiation that results in photochemical darkening. Originally it was assumed that lifetime increases uniformly with increasing transparency. We now have cases where materials with very different absorbances (TAFx4P and 46P) have similar lifetimes and materials with similar absorptions (TAFx46P and 2P) have very different lifetimes. These findings demonstrate the importance of the relative quantum efficiencies as the $157 \mathrm{~nm}$ light energy distributes itself along degradative versus non-degradative pathways. In an effort to identify chemical and structural features that control lifetime, we have been studying model molecular materials, some quite similar to the monomer units used to make our pellicle candidates. Several of these models have shown transparencies much higher and lifetimes far longer than our best pellicle candidates to date.

\section{INTRODUCTION}

With the introduction of $157 \mathrm{~nm}$ as the next optical lithography wavelength, the need for new pellicle materials optimized for this wavelength has produced much activity in development of ultra transparent fluoropolymers suitable for $157 \mathrm{~nm}$ pellicle applications where absorbances below $0.03 / \mu \mathrm{m}$ are necessary to achieve pellicle transmissions above $98 \%$ ${ }^{1}$ and which exhibit a sufficient lifetime under $157 \mathrm{~nm}$ irradiation. ${ }^{2}$

In the development of a viable $157 \mathrm{~nm}$ soft pellicle were have pursued an extensive and comprehensive materials development program. The different stages of this effort are shown in Figure 1, where we show the development stages in yellow and the testing stages in gray. We discuss the effects of extrinsic factors in another paper in this meeting. ${ }^{3}$

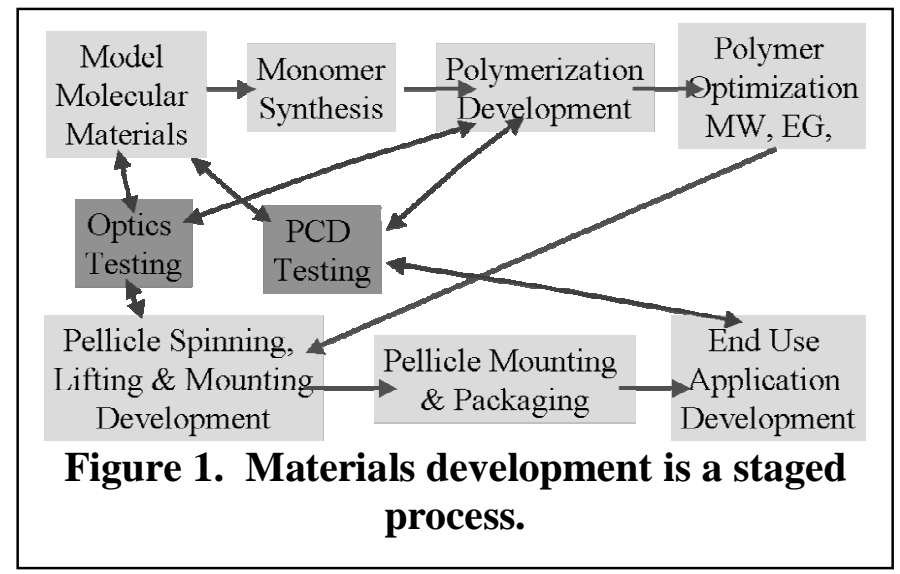

It is important to keep in mind these different stages in the development as results are being presented. In the case where a new monomer is used to produce a new polymer, this polymer may have very desirable optical absorption, for example, but it may not be possible to optimize its molecular weight to such a degree that that polymer can be used to spin an unsupported polymer membrane. Still the finding of a polymer with that level of optical absorption, can be used to inform the future development of other classes of polymers which can be used to produce pellicle membranes.

We have also added the study of model molecular materials as a source of chemical insights for 
fluoromonomer development. The second set of molecular material results are presented in section 4 .

\section{ABSORBANCE AND PCD TESTING ON NEW PELLICLE POLYMERS}

New monomers have been used in the production of new TAFx polymers, TAFx31P to TAFx 58P. These new polymer families represent the output of our monomer design effort, and the properties of these new TAFx polymers are presented here.

For reference, in Table 1, we list the absorbance of a representative sample of each of the experimental TAFx materials synthesized to date. These polymer families in some cases have had 30 or more polymerization runs performed while optimizing the materials properties.

\section{Measured TAFx3P Pellicle Transmission}

With continuing optimization of the polymer materials and the pellicle spinning process we have continued to increase the $157 \mathrm{~nm}$ transmission of our pellicles. In Figure 2 we show the transmission of a TAFx3P-10665 pellicle which exhibits a peak transmission of $98.6 \%$ at $155 \mathrm{~nm}$, our best result to date. The transmission of this pellicle at $157.6 \mathrm{~nm}$ ( not at a fringe peak) is $95.9 \%$ where these measurements are made with a $0.92 \mathrm{~nm}$ spectrophotometer band pass.

\section{0\% PCD Lifetime}

For radiation durability it is useful to define a lifetime for the material. We define this for a given PCD rate, or value of $\mathrm{Ai} / \mathrm{um} / \mathrm{J}$, for a sample of 0.8 micrometer thickness, how many Joules $/ \mathrm{cm}^{2}$ are required for the initial transmission of the sample to drop an additional $10 \%$. We therefore refer to this as the $10 \%$ PCD Lifetime (or $\mathrm{L}_{10}$ ), in units of Joules $/ \mathrm{cm}^{2}$ dose of $157 \mathrm{~nm}$ radiation. Note that these lifetimes are based on a linear model for PCD processes. Still they serve to capture changes in the behavior of materials on a comparative basis. An increase in the 10\% PCD lifetime corresponds to increased radiation durability.

Lifetime $=\frac{A_{\cdot_{i}}}{P C D \text { rate }}=\frac{\log _{10}\left(T_{\text {init }} /\left(T_{\text {init }}-\Delta T\right)\right)}{t \text { PCD rate }}$

\section{TAFx46P \& 48P: Polymers From New Designer Monomer}

TAFx46P exhibits the longest lifetime $\left(\mathrm{L}_{10}=\right.$ $6.6 \mathrm{~J} / \mathrm{cm}^{2}$ ), when tested under $1 \mathrm{ppm} \mathrm{O}_{2}$, of any polymer to date. This is a very interesting results since it also has a relatively high absorbance $(0.025 / \mu \mathrm{m})$ when compared to, for example, TAFx3P, and also exhibits and absorbance peak at $170 \mathrm{~nm}$. TAFx $48 \mathrm{P}$ has a similar comonomer, but has a lower absorbance $(0.007 / \mu \mathrm{m})$, and therefore may be expected by our $1^{\text {st }}$ design paradigm (lifetime is proportional to absorbance) as having a yet longer lifetime than TAFx46P. Upon PCD testing it is found that TAFx 48P has a 10\% PCD lifetime of only $1.8 \mathrm{~J} / \mathrm{cm}^{2}$.

PCD and 10\% Lifetime of TAFx46P and TAFx4P

Upon performing PCD tests on TAFx46P and TAFx4P we find that they both exhibit long $10 \%$ PCD

\begin{tabular}{|c|c|c|c|c|c|c|}
\hline TAFx & $\mathbf{A} / \boldsymbol{\mu}$ & $\mathbf{T A F x}$ & $\mathbf{A} / \boldsymbol{\mu}$ & $\mathbf{T A F x}$ & $\mathbf{A} / \boldsymbol{\mu}$ \\
\hline $1 \mathrm{P}$ & 0.012 & $21 \mathrm{P}$ & 0.006 & $50 \mathrm{P}$ & $>5$ \\
\hline $2 \mathrm{P}$ & 0.014 & $22 \mathrm{P}$ & 0.7 & $51 \mathrm{P}$ & 3.6 \\
\hline $3 \mathrm{P}$ & 0.009 & $23 \mathrm{P}$ & 1.37 & $52 \mathrm{P}$ & $>4$ \\
\hline $4 \mathrm{P}$ & 0.015 & $24 \mathrm{P}$ & 0.007 & $53 \mathrm{P}$ & 0.035 \\
\hline $5 \mathrm{P}$ & 0.015 & $25 \mathrm{P}$ & 0.43 & $56 \mathrm{P}$ & $<0.006$ \\
\hline $6 \mathrm{P}$ & 0.03 & $26 \mathrm{P}$ & 0.63 & $57 \mathrm{P}$ & 0.035 \\
\hline $7 \mathrm{P}$ & 0.016 & $27 \mathrm{P}$ & 0.008 & $58 \mathrm{P}$ & $<0.006$ \\
\hline $8 \mathrm{P}$ & 0.13 & $28 \mathrm{P}$ & 0.603 & $100 \mathrm{P}$ & 1.9 \\
\hline $9 \mathrm{P}$ & 0.21 & $29 \mathrm{P}$ & 0.085 & & \\
\hline $10 \mathrm{P}$ & 0.35 & $30 \mathrm{P}$ & 0.104 & & \\
\hline $11 \mathrm{P}$ & 0.38 & $31 \mathrm{P}$ & $<0.006$ & & \\
\hline $12 \mathrm{P}$ & 0.93 & $33 \mathrm{P}$ & 0.15 & & \\
\hline $13 \mathrm{P}$ & 1.335 & $36 \mathrm{P}$ & 0.006 & & \\
\hline $14 \mathrm{P}$ & 1.367 & $37 \mathrm{P}$ & 0.99 & & \\
\hline $15 \mathrm{P}$ & 1.44 & $41 \mathrm{P}$ & 2.0 & & \\
\hline $16 \mathrm{P}$ & 3.9 & $42 \mathrm{P}$ & 1.93 & & \\
\hline $17 \mathrm{P}$ & 5.284 & $43 \mathrm{P}$ & 0.404 & & \\
\hline $18 \mathrm{P}$ & 5.6 & $44 \mathrm{P}$ & 0.131 & & \\
\hline $19 \mathrm{P}$ & 0.034 & $46 \mathrm{P}$ & 0.026 & & \\
\hline $20 \mathrm{P}$ & 0.028 & $48 \mathrm{P}$ & 0.006 & & \\
\hline $\mathrm{Tab}$ & $1.2 \mathrm{Abs}$ & $\mathrm{bances}$ & $\mathbf{0 f} 0 \mathrm{sam}$ \\
\hline
\end{tabular}

Table 1. Absorbances of one sample of each TAFx Polymer Family

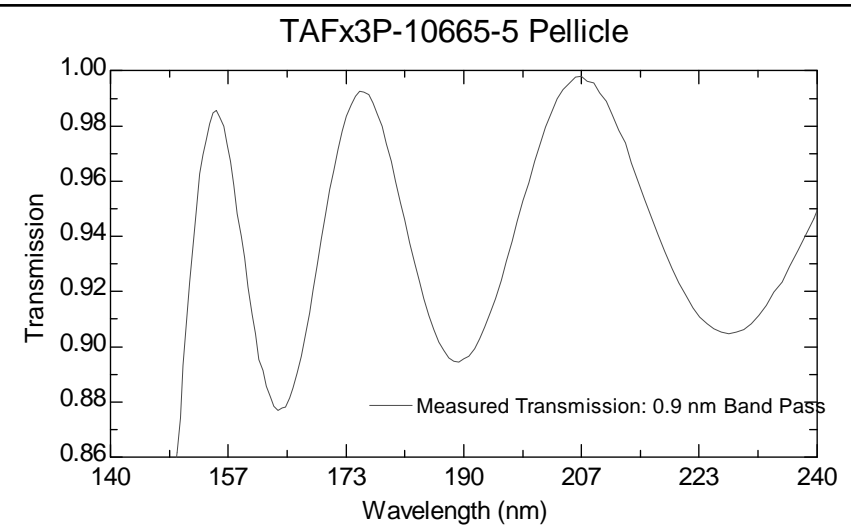

Figure 2. Spectral transmission of Pellicle TAFx3P-10665-6 . 
lifetimes when tested under $1 \mathrm{ppm} \mathrm{O}_{2}$. These results are shown in Figure 3 with comparison to TAFx2P and TAFx3P polymers

In Figure 4 we show the 10\% PCD lifetimes for many of the polymers measured to date. Overall for any one polymer, under identical testing conditions, there is substantial variability in the $10 \%$ lifetime, which suggests the presence of uncontrolled variables in the testing and materials.

The TAFx2P polymer $10 \%$ lifetimes are all below $3 \mathrm{~J} / \mathrm{cm}^{2}$, and the TAFx3P polymer lifetimes are below 4 Joules $/ \mathrm{cm}^{2}$ when tested under $1 \mathrm{ppm} \mathrm{O}_{2}$ environment. We can also see that the TAFx46P polymer and also a TAFx4P polymer both show noticeably longer lifetimes, with the TAFx46P showing a 6.6 Joule $/ \mathrm{cm}^{2} 10 \%$ lifetime.

\section{Correlation of PCD}

\section{Lifetime and Optical}

Absorbance

One of the design paradigms in this project has been that the 10\% PCD lifetime will be proportional to the optical absorbance of the polymer. This is obviously true for very strongly absorbing polymers, but as is shown in Figure 5, once the optical absorbance is sufficiently low, the lifetime is not simply correlated to absorbance. This is to say that the details of the photochemistry, and the quantum efficiencies of different photochemical pathways in different polymers will play the controlling role in determining the $10 \%$ lifetime, and the best example of this is in TAFx46P which is four times more absorbing than TAFx3P, yet has a 50\% longer 10\% lifetime. Another example is seen in TAFx46P and 48P where a small change in one comonomer drops the absorbance by a factor of three, while the lifetime is also reduced by a factor of three.

\section{NANOMECHANICS AND POLYMER IRRADIATION}

The NanoScratch Tester ${ }^{4}$ (NST) is a sensitive nanomechanical device with an integrated Atomic Force Microscope for high resolution 3-dimensional imaging. This new instrument developed in a collaboration between DuPont Performance 
Coatings, DuPont Central Research and Development, and CSEM Instruments makes it possible to measure mechanical properties such as viscoelastic recovery, plastic yield and fracture on very small amounts of material. Because the NST can apply and measure forces down to tens of microNewtons, and has 3-dimensional spatial resolution in the nanometer range, it is also among the world's best profilometers. The nanoscratch tester determines quantitative load displacement curves and both normal an lateral forces on the indenter while doing

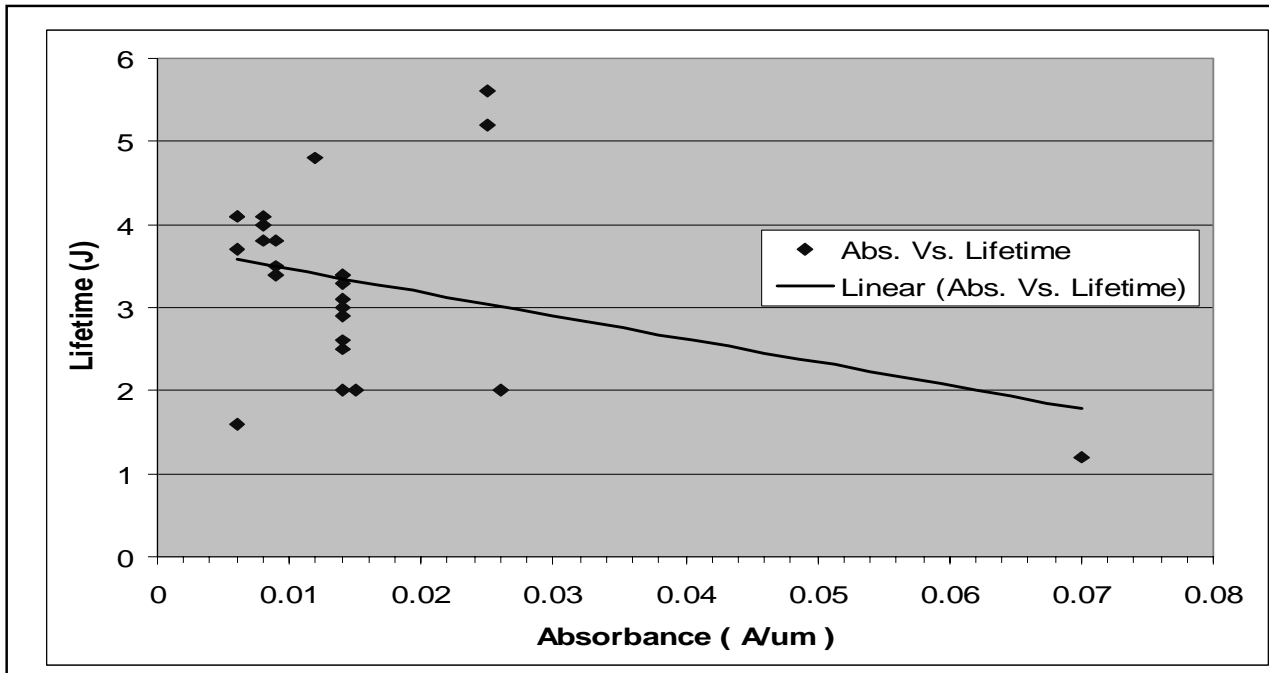

Figure 5. Correlation plot of lifetime and absorbance.

nanoindentations and also nanoscratches (as shown in Figure 6).

Nanoindentation Results

We took a 1 micron thick sample of TAFx3P on a silicon wafer, and irradiated one region of the sample to a dose of $45 \mathrm{~J} / \mathrm{cm}^{2}$. We then performed the nanoindentation studies.

For nanoindentation studies one uses a three sided pyramidal nanoindenter and applies a small normal force. The force used in the present work was 0.5 milliNewtons $(0.5 \mathrm{mN})$ in magnitude. For the results to be representative of the properties of a thin film on a substrate we want the penetration into the polymer membrane to not go beyond $20 \%$ of the film thickness. Two typical nanoindents are shown in Figure 7. The indent, under constant load, into the $157 \mathrm{~nm}$ laser irradiated polymer (right side) is smaller and less deep. During the nanoindentation we can measure the load displacement curve, and these are shown in Figure 8 for the unirradiated TAFx3P polymer and the $157 \mathrm{~nm}$ laser irradiated region of the sample where we overlay the load displacement curves for comparison. For each fixed applied load up to $0.5 \mathrm{mN}$, the indenter penetrates less deeply in the irradiated case, showing that the irradiated area has become harder.

We can analyze the load displacement curves to determine the Hardness $(\mathrm{H})$, the Vickers Hardness $(\mathrm{Hv})$ and the Young's Modulus (E) in the unirradiated and irradiated TAFx3P polymer. We find that the Young's modulus of the polymer is unaffected by irradiation, while the Hardness of the irradiated polymer is increased.

\section{ABSORP TION \\ AND \\ PHOTOC \\ HEMICA \\ $\mathbf{L}$ \\ DARKEN \\ ING OF \\ MOLEC \\ ULAR \\ MATERI \\ ALS}

Molecular

materials are being looked at as model compounds.

This work has four major goals

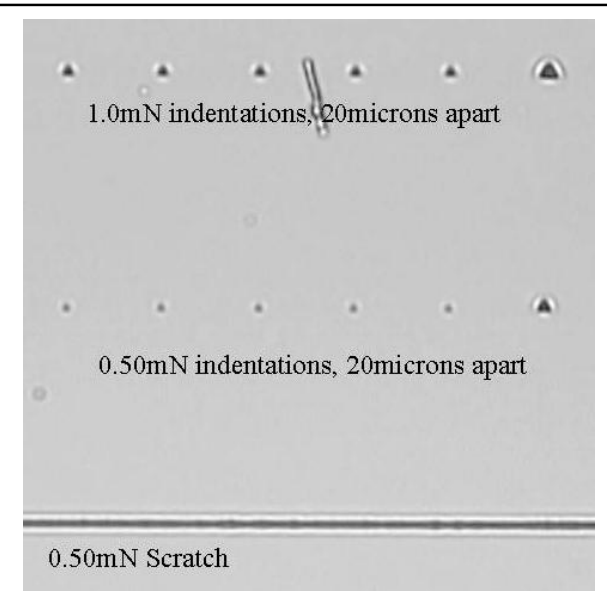

Figure 6. Optical micrograph of nanoindentations and scratches on TAFx3P pellicle polymer in the irradiated area.

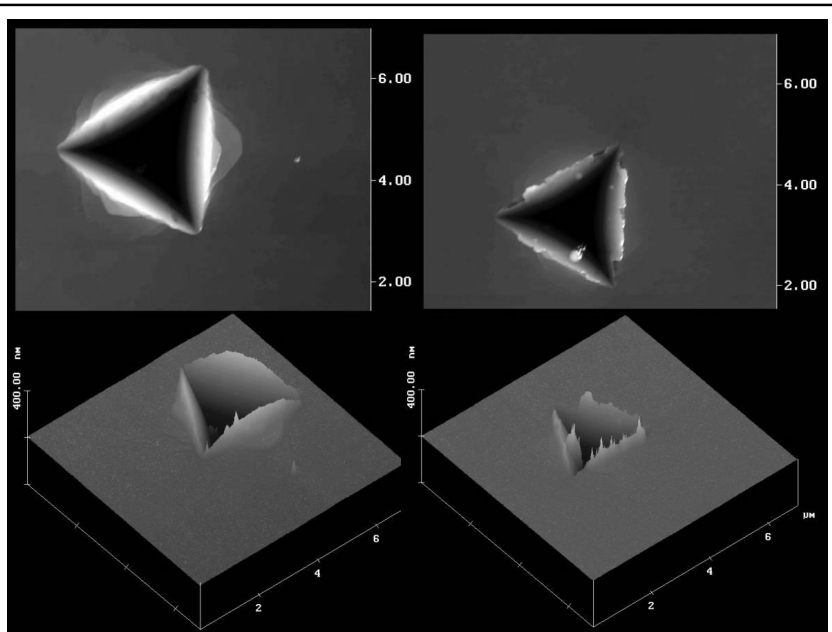

Figure 7. Nanoindentations taken with a 0.5 $\mathrm{mN}$ load in the unirradiated polymer (left side) and irradiated area (right side). 
. Our first goal is to find simple organic compounds having a much higher transparency at $157 \mathrm{~nm}$ than our best pellicle candidates $(\mathrm{A} / \mu \sim 0.006$ to 0.01$)$. The existence of highly transparent organics encourages the belief that similarly transparent polymers could be found. Our second goal is to demonstrate that having such high transparencies could produce useful extensions of $157 \mathrm{~nm}$ lifetime. Certainly just showing that highly transparent model compounds photodegrade much less rapidly than our pellicle candidates is encouraging. Our third goal is to use the model compounds for mechanistic studies of photodegradation. Unlike polymers, the model compounds have sufficiently simple structures that the chemical details of photodegradation can likely be followed with great precision. Our fourth goal would be to develop correlations between model compound structure and transparency/lifetime as a guide for designing better pellicle polymers.

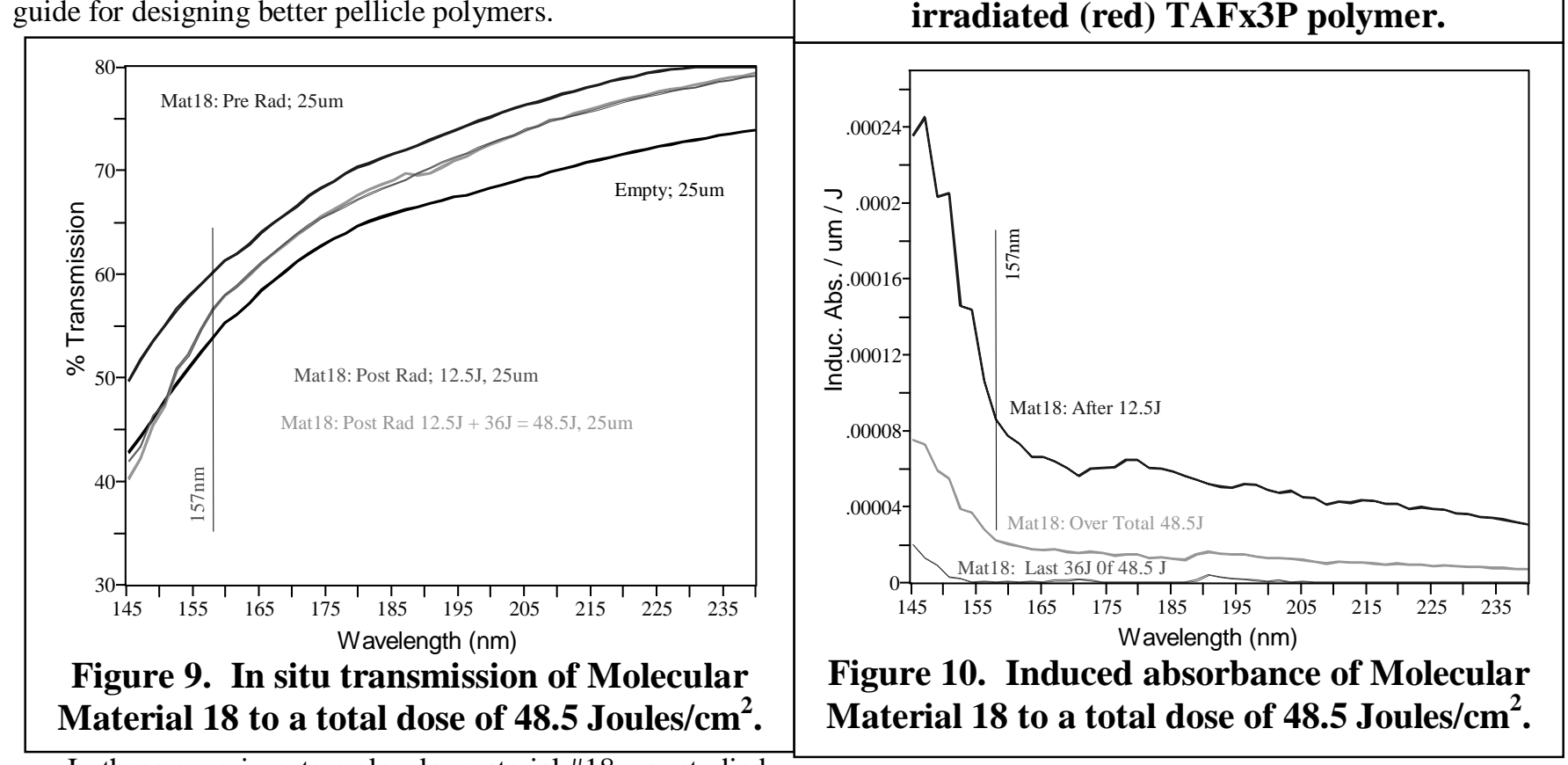

In these experiments molecular material \#18 was studied

in a cell with a thickness of 25 microns, thereby giving us very good sensitivity to the optical absorbance and to the $157 \mathrm{~nm}$ laser radiation induced absorbance. The $157 \mathrm{~nm}$ laser dose rate was $3 \mathrm{~J} /$ minute, and the energy density was $\sim 1 \mathrm{~mJ} / \mathrm{cm}^{2}$. Transmission was measured as a function of total dose of $157 \mathrm{~nm}$ laser radiation. We also measured the spectral transmission after varying time delays. We use these spectra to calculate the induced absorbance /micron thickness/Joule dose. These calculations are done using two spectral transmission files, and therefore, in an experiment where the cell is exposed to two sequential doses of $157 \mathrm{~nm}$ laser radiation, we can calculate the induced absorbance over the initial dose, the final total (the second exposure) or over the total dose (combining the initial and final doses). We also remeasured the spectral transmission after a time delay and report subsequent changes in the induced absorption. We observed both laser induced photochemical darkening (PCD) and photochemical bleaching (PCB). The discovery of PCB is a very exciting finding.

Molecular Material 18 Irradiated To $48.5 \mathrm{Joule} / \mathrm{cm}^{2}$ Total Dose

In Figure 9 we show the spectral transmission for molecular material 18 pre radiation, then after $12.5 \mathrm{~J} / \mathrm{cm}^{2}$ initial dose, and then after an additional $36 \mathrm{Joule} / \mathrm{cm}^{2}$ final dose to a total dose of $48.5 \mathrm{Joules} / \mathrm{cm}^{2}$. In Figure 10 we then show the induced absorbance / um / J for the initial $12.5 \mathrm{~J} / \mathrm{cm}^{2}$ dose, for the final $36 \mathrm{Joule} / \mathrm{cm}^{2}$ dose and over the total $48.5 \mathrm{~J} / \mathrm{cm}^{2} \mathrm{dose}$. After the initial $12.5 \mathrm{Joule} / \mathrm{cm}^{2}$ dose the transmission decreases and we see a $10 \%$ lifetime of $475 \mathrm{Joules} / \mathrm{cm}^{2}$. Upon irradiation for an additional $36 \mathrm{Joules} / \mathrm{cm}^{2}$, there is negligible change in the transmission or the induced absorbance and we find a $300,000 \mathrm{Joule} / \mathrm{cm}^{2} 10 \%$ lifetime. If we calculate over the whole $48.5 \mathrm{Joule} / \mathrm{cm}^{2}$ dose, the $10 \%$ lifetime is 1,767 Joules $/ \mathrm{cm}^{2}$. 


\section{Molecular Material 18 Irradiated To $113 \mathrm{Joule} / \mathrm{cm}^{2}$ Total Dose}

In Figure 11 we show the spectral transmission for molecular material 18 pre radiation, then after $25.4 \mathrm{~J} / \mathrm{cm}^{2}$ initial dose, and then after an additional $87.5 \mathrm{Joule} / \mathrm{cm}^{2}$ final dose to a total dose of $113 \mathrm{Joules} / \mathrm{cm}^{2}$, the transmission is then remeasured after a time delay of 60 hours. In Figure 12 we then show the induced absorbance $/ \mathrm{um} / \mathrm{J} / \mathrm{cm}^{2}$ for the initial 25.4 $\mathrm{J} / \mathrm{cm}^{2}$ dose, for the final $87.5 \mathrm{Joule} / \mathrm{cm}^{2}$ dose and over the total $113 \mathrm{~J} / \mathrm{cm}^{2}$ dose, and then after the 60 hour time delay.

After the initial 25.4 Joule $/ \mathrm{cm}^{2}$ dose we calculate at $10 \%$ lifetime of $868 \mathrm{Joules} / \mathrm{cm}^{2}$. Over the final $87.4 \mathrm{Joules} / \mathrm{cm}^{2}$ the transmission drop is much reduced and the induced absorption is much smaller than the changes over the initial dose, and the $10 \%$ lifetime is $16,214 \mathrm{Joules} / \mathrm{cm}^{2}$. Over the total $113 \mathrm{Joule} / \mathrm{cm}^{2}$ dose, the $10 \%$ lifetime is $3,270 \mathrm{Joules} / \mathrm{cm}^{2}$.

After the final dose to a total 113 Joules $/ \mathrm{cm}^{2}$, we then waited 60 hours and remeasured the spectral transmission of the cell and calculated the induced absorbance and the $10 \%$ lifetime. Here we see the occurrence of time delayed PCD, with the transmission further decreasing after this 60 hour time delay, and the $10 \%$ lifetime decreasing from 3,270 to 1,108 Joules $/ \mathrm{cm}^{2}$.

\section{Molecular Material 18 Irradiated To $25 \mathrm{Joule} / \mathrm{cm}^{2}$ Total Dose}

In Figure 13 we show the spectral transmission for molecular material 18 pre radiation, then after 12.75 $\mathrm{J} / \mathrm{cm}^{2}$ initial dose, then remeasured after a 16 hour delay, and then after an additional $14.25 \mathrm{Joule} / \mathrm{cm}^{2}$ final dose to a total dose of 27 Joules $/ \mathrm{cm}^{2}$, the transmission is then remeasured after a time delay of 60 hours. In Figure 14 we then show the induced absorbance / um / J for the initial $12.75 \mathrm{~J} / \mathrm{cm}^{2}$ dose, after the 16 hour delay and then for the final $14.25 \mathrm{Joule} / \mathrm{cm}^{2}$ dose and over the total $27 \mathrm{~J} / \mathrm{cm}^{2}$ dose, and then after the 60 hour time delay.

In these measurements we expose at a rate of 3 Joules total dose per minute, so that the exposure time of the molecular material is short compared to the 16 or 60 hour time delays for remeasurement of the spectral transmission.

After the initial dose of $12.75 \mathrm{Joules} / \mathrm{cm}^{2}$, we calculate a $10 \%$ lifetime of $569 \mathrm{Joules} / \mathrm{cm}^{2}$, again comparable to our previous results. We then check for time delayed PCD by waiting 16 hours and remeasuring the cell, and we find the transmission has decreased, and the $10 \%$ lifetime has been reduced to $86 \mathrm{Joules} / \mathrm{cm}^{2}$. We

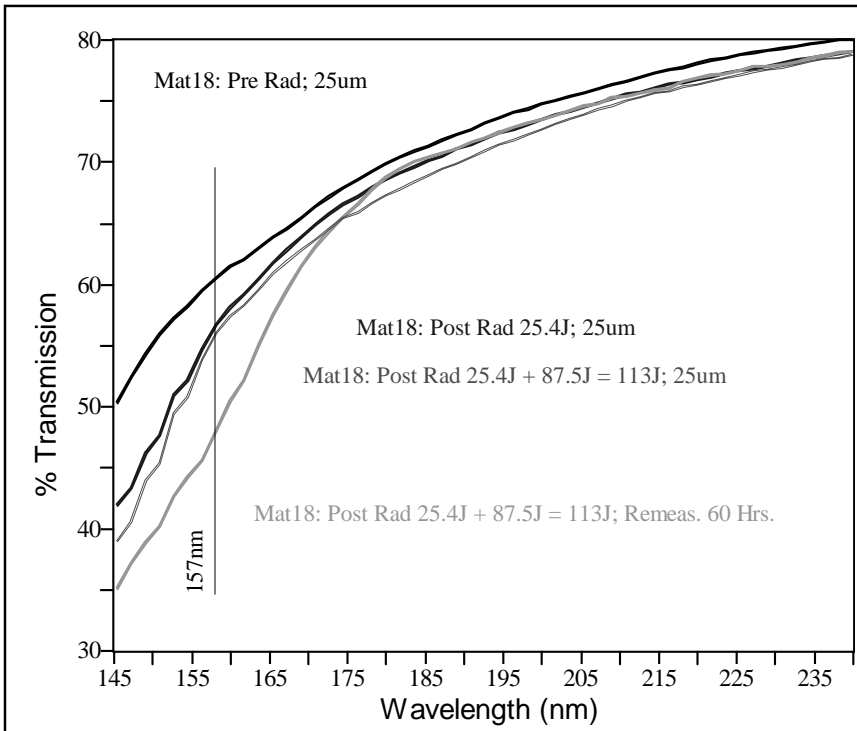

Figure 11. In situ transmission of Molecular Material 18 to a total dose of $113 \mathrm{Joules} / \mathrm{cm}^{2}$.

\begin{tabular}{|c|c|c|c|}
\hline $\begin{array}{l}\text { Induc } \\
\text { Abs / } \\
\text { um / J } \\
\end{array}$ & $\begin{array}{l}\text { Induc } \\
\text { Abs / um } \\
\text { / J }\end{array}$ & Dose J & $\begin{array}{l}\text { 10\% Life }(\mathrm{J}) \\
\text { From Induc } \\
\text { Abs. }\end{array}$ \\
\hline s2992j & & Initial 12.5J & 457 \\
\hline s2993j & 1.3E-07 & Final 36J & 311,414 \\
\hline s2994j & $2.2 E-05$ & Total 48.5J & 1,767 \\
\hline s3002j & 4.5E-05 & Initial 25.4J & 868 \\
\hline s3003j & $2.4 \mathrm{E}-06$ & Final 87.5J & 16,214 \\
\hline s3004j & 1.2E-05 & Total 113J & 3,270 \\
\hline s3005j & $3.5 E-05$ & $113 \mathrm{~J}+60 \mathrm{hrs}$ & 1,108 \\
\hline s3012j & 6.9E-05 & Initial 12.75J & 569 \\
\hline s3013j & $4.5 E-04$ & $12.75 \mathrm{~J}+16 \mathrm{hrs}$ & 86 \\
\hline s3014j & $-2.6 \mathrm{E}-04$ & Final 12.25J & -151 \\
\hline s3015j & 1.3E-04 & Total 25 & 306 \\
\hline
\end{tabular}

Table 2. 10\% Lifetimes calculated for Molecular Material 18.

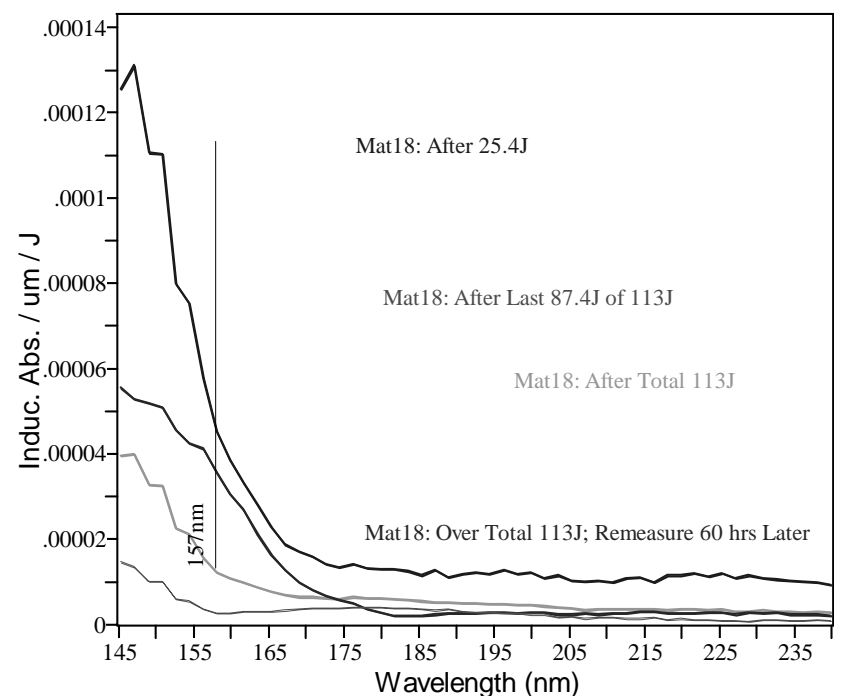

Figure 12. Induced absorbance of Molecular Material 18 to a total dose of $113 \mathrm{Joules} / \mathrm{cm}^{2}$. 
then impose the final dose of radiation, an additional $14.25 \mathrm{Joules} / \mathrm{cm}^{2}$ and we find that the spectral transmission increases, corresponding to a bleaching of the absorption of the material by the laser. This PCB produces an effective negative 10\% lifetime of $-115 \mathrm{Joules} / \mathrm{cm}^{2}$. Over the total $25 \mathrm{Joule} / \mathrm{cm}^{2}$ dose, with the included 16 hour time delay we find a $10 \%$ lifetime of 306 Joules $/ \mathrm{cm}^{2}$.

\section{0\% Lifetimes of Molecular Material 18}

We summarize the calculated $10 \%$ lifetimes for these experiments on molecular material 18 in Table2.

\section{CONCLUSIONS}

We have continued with our synthesis and characterization of pellicle candidate polymers, using transparency and photochemical darkening rate as the primary screens. New candidates with absorbances $\mathrm{A} / \mu<0.01$ and with photochemical darkening rates, measured under $1 \mathrm{ppm} \mathrm{O}_{2}$, up to 6.6 joules $/ \mathrm{cm}^{2}$ have been identified and added to our list of candidates that meet the basic transparency specification $\left(98 \%\right.$ ) but fail on the lifetime specification (ideally $7.5 \mathrm{Kjoules} / \mathrm{cm}^{2}$ ).

\section{TAFx46P: Optical Absorbance and Lifetime}

New polymers continue to be synthesized and tested for transparency and lifetime. TAFx46P and 48P explores the connection between transparency and lifetime for pellicle candidates at $157 \mathrm{~nm}$. The starting point here is TAFx $46 \mathrm{P}$ which has the longest lifetime thus far $\left(6.6 \mathrm{Joules} / \mathrm{cm}^{2}, \mathrm{~N}_{2}\right.$ with $1 \mathrm{ppm}$ of $\left.\mathrm{O}_{2}\right)$ in spite of a relatively high absorption $(\mathrm{A} / \mu=0.026)$. Changing just one bond in TAFx46P increases the transparency $\geq 2.6 \mathrm{X}$, but surprisingly decreased lifetime $\sim 50 \%$. This challenges our fundamental assumption that the more transparent a polymer the longer its lifetime. Once a certain level of transparency has been achieved, the quantum yields for photodegradation apparently can be as important or even more important to lifetime than the fraction of incident light absorbed. This points up a need for a more fundamental understanding of what chemical structures are actually absorbing the light and how this absorbed light energy redistributes itself between damaging chemical change and harmless release to the environment. A good place to start might be the photochemical degradation products of simple compounds.

\section{Nanoindentation Summary}

A nanoindentation probe of the Nanoscratch Tester has been used to qualitatively compare the mechanical properties of exposed and unexposed areas of TAFx3P films. Exposure to $157 \mathrm{~nm}$ light makes the TAFx3P film stiffer (increased hardness) and more brittle when indented and more subject to cracking when scratched, physical property changes suggestive of crosslinking. Samples of TAFx2P and TAFx4P have now been submitted to determine whether most pellicle candidates respond similarly.

\section{Molecular Materials Summary}

Tests on molecular material \#18 have demonstrated experimentally, with total doses up to $113 \mathrm{Joules} / \mathrm{cm}^{2}$, that fluoromolecules can withstand 157nm laser irradiation and achieve 10\% PCD lifetimes (lifetimes for a 10\% transmission drop in a 0.8 micron thick sample) in the range from 500 to 3000 Joules $/ \mathrm{cm}^{2}$.

The initial PCD rate, during say the initial 10 or 20 joules of dose is much higher than the PCD rate over longer

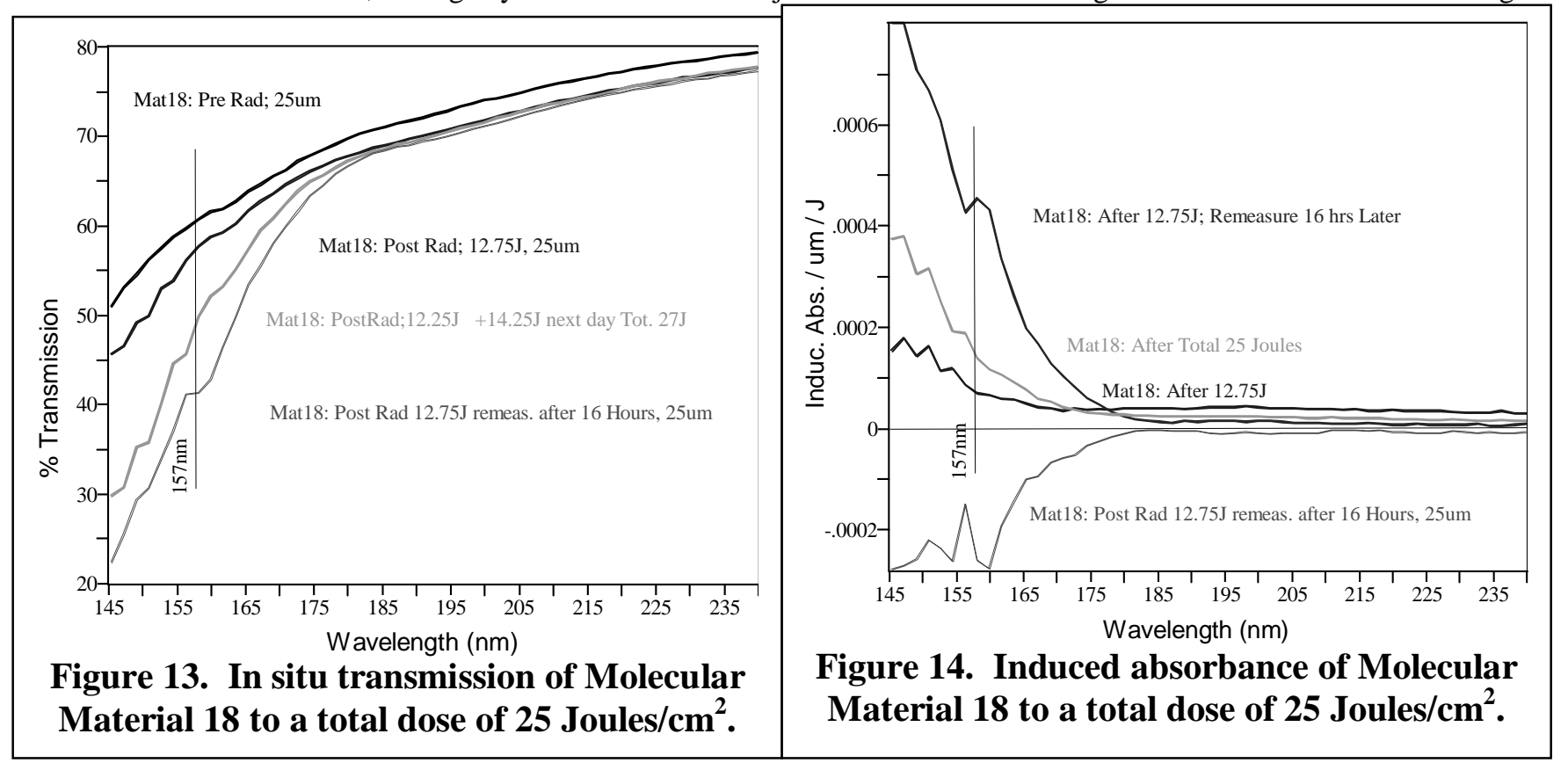


doses, so that after the initial photochemical darkening, the darkening rate is decreased, and the $10 \%$ lifetimes are increased. This is dramatically different from what has been seen to date in polymer on $\mathrm{CaF}_{2}$ or pellicle samples, where PCD is an exponential run away process. Molecular material 18 shows a sublinear PCD process where the darkening rate decreases with increasing dose.

We have also seen time delayed PCD, where in the hours after irradiation stops, darkening continues, and this can give us insight into the photochemistry of PCD in these materials.

We also observe Photochemical Bleaching (PCB), in which for the first time upon $157 \mathrm{~nm}$ laser irradiation the material shows increasing transmission and negative induced absorbance.

\section{ACKNOWLEDGEMENTS}

The Lincoln Laboratory portion of this work was performed under a Cooperative Research and Development Agreement between MIT Lincoln Laboratory and SEMATECH. Opinions, interpretations, conclusions, and recommendations are those of the authors and are not necessarily endorsed by the United States Government

\section{REFERENCES}

${ }^{1}$ R. H. French, R. C. Wheland, D. J. Jones, J. N. Hilfiker, R. A. Synowicki, F. C. Zumsteg, J. Feldman, A. E. Feiring, "Fluoropolymers for 157nm Lithography: Optical Properties from VUV Absorbance and Ellipsometry Measurements", Optical Microlithography XIII, SPIE Vol. 4000, edited by C. J. Progler, 1491-1502 (2000).

${ }^{2}$ Roger H. French, Joseph Gordon, David J. Jones, M. F. Lemon, Robert C. Wheland, Edward Zhang, Fredrick C. Zumsteg, Kenneth G. Sharp, Weiming Qiu, "Materials Design and Development of Fluoropolymers for Use as Pellicles in 157nm Photolithography", Optical Microlithography XIV, SPIE Vol. 4346, (2001).

${ }^{3}$ V. Liberman, M. Rothschild, J. H. C. Sedlacek, A. Grenville, R. H. French, "Behavior of Candidate Organic Pellicle Materials Under 157-nm Laser Irradiation”, 2002 SPIE Meeting "Optical Microlithography XV”, March 2002. SPIE Vol. 4691-56.

${ }^{4}$ http://www.microphotonics.com/nst.html or http://instruments.csem.ch/ 